\title{
Chinese expert consensus on the prevention of abdominal pelvic adhesions after gynecological tumor surgeries
}

\author{
Jinghe Lang ${ }^{1}$, Ding $\mathrm{Ma}^{2}$, Yang Xiang ${ }^{1}$, Keqin Hua ${ }^{3}$, Kaijiang Liu ${ }^{4}$, Lingya Pan ${ }^{1}$, Ping Wang ${ }^{5}$, \\ Shuzhong Yao ${ }^{6}$, Fujie Zhao ${ }^{7}$, Wenjun Cheng ${ }^{8}$, Manhua Cui ${ }^{9}$, Hongyan Guo ${ }^{10}$, Ruixia Guo ${ }^{11}$, Li Hong ${ }^{12}$, \\ Peiling Li ${ }^{13}$, Mubiao Liu ${ }^{14}$, Yuanguang Meng ${ }^{15}$, Hui Wang ${ }^{2}$, Jianliu Wang ${ }^{16}$, Wuliang Wang ${ }^{17}$, Ming Wu ${ }^{1}$, \\ Xingsheng Yang ${ }^{18}$, Jun Zhang ${ }^{19}$
}

${ }^{1}$ Department of Genecology and Obstetrics, Beijing Union Medical College Hospital, Beijing 100140, China; ${ }^{2}$ Department of Genecology and Obstetrics, Tongji Hospital, Tongji Medical College, Huazhong University of Science and Technology, Wuhan 430030, China; ${ }^{3}$ Department of Genecology and Obstetrics, Fudan University Affiliated Obstetrics and Gynecology Hospital, Shanghai 200032, China; ${ }^{4}$ Department of Genecology and Obstetrics, Renji Hospital, Shanghai Jiao Tong University School of Medicine, Shanghai 200001, China; ${ }^{5}$ Department of Genecology and Obstetrics, West China Second University Hospital, Sichuan University, Chengdu 610041, China; ${ }^{6}$ Department of Genecology and Obstetrics, The First Affiliated Hospital of Zhongshan University, Guangzhou 510080, China; ${ }^{7}$ Department of Genecology and Obstetrics, Shengjing Hospital Affiliated with China Medical University, Shenyang 110004, China; ${ }^{8}$ Department of Genecology and Obstetrics, Jiangsu Provincial People's Hospital, Nanjing 210036, China; ${ }^{9}$ Department of Genecology and Obstetrics, Second Hospital of Jilin University, Changchun 130042, China; ${ }^{10}$ Department of Genecology and Obstetrics, Third Hospital of Peking University, Beijing 100191, China; ${ }^{11}$ Department of Genecology and Obstetrics, The First Affiliated Hospital of Zhengzhou University, Zhengzhou 450052, China; ${ }^{12}$ Department of Genecology and Obstetrics, Wuhan University People's Hospital, Wuhan 430060, China; ${ }^{13}$ Department of Genecology and Obstetrics, The Second Affiliated Hospital of Harbin Medical University, Harbin 150001, China; ${ }^{14}$ Department of Genecology and Obstetrics, Guangdong Provincial People’s Hospital, Guangzhou 510080, China; ${ }^{15}$ Department of Genecology and Obstetrics, Chinese People's Liberation Army General Hospital, Beijing 100853, China; ${ }^{16}$ Department of Genecology and Obstetrics, Peking University People's Hospital, Beijing 100044, China; ${ }^{17}$ Department of Genecology and Obstetrics, The Second Affiliated Hospital, Zhengzhou University, Zhengzhou 450052, China; ${ }^{18}$ Department of Genecology and Obstetrics, Qilu Hospital of Shandong University, Jinan 250012, China; ${ }^{19}$ Department of Genecology and Obstetrics, Beijing Anzhen Hospital, Capital Medical University, Beijing 100011, China Correspondence to: Ding Ma. Department of Genecology and Obstetrics, Tongji Hospital, Tongji Medical College, Huazhong University of Science and Technology, Wuhan 430030, China. Email: dma@tjh.tjmu.edu.cn; Jinghe Lang. Department of Genecology and Obstetrics, Beijing Union Medical College Hospital, Beijing 100140, China. Email: langjh@hotmail.com.

\begin{abstract}
Adhesion is a postoperative complication that has plagued gynecologists for many years, as $60-90 \%$ of gynecological patients develop adhesions after abdominopelvic surgeries. Abdominopelvic adhesions could lead to chronic pelvic pain, infertility, intestinal obstruction, and complicated reoperations. Adhesions might also increase the risk of postoperative chemoradiotherapy failure and endanger patients' lives, especially after surgeries for gynecological malignant tumors. The aim of this consensus was to review the pathogenesis and clinical consequences of adhesions and to summarize various surgical procedures and preventive measures that can reduce the occurrence of adhesions after gynecological tumor surgeries based on a discussion among well-known domestic gynecology specialists.
\end{abstract}

Keywords: Adhesion; tumor surgery; prevention; expert consensus

Submitted Dec 02, 2019. Accepted for publication Feb 10, 2020.

doi: 10.21037/atm.2020.02.53

View this article at: http://dx.doi.org/10.21037/atm.2020.02.53 


\section{Introduction}

Adhesion refers to the connection of separated tissues by fibrous bands under normal circumstances. Adhesions occur in $60 \%$ to $90 \%$ of patients after abdominopelvic surgeries (1) and are the most common surgical complication. Abdominopelvic adhesions can lead to chronic pelvic pain, infertility, intestinal obstruction, and complicated reoperations (2-4). Adhesions after gynecological tumor surgeries also increase the risk of postoperative chemoradiotherapy failure and endanger patients' lives.

\section{The effect of adhesion on the treatment of gynecological tumors}

Adhesions are the most common cause of intestinal obstruction. More than $30 \%$ of colonic obstructions and $80 \%$ of small bowel obstructions, which have mortality rates of $3-10 \%$, are caused by adhesions. Moreover, $48 \%$ of chronic pelvic pain and $20-40 \%$ of infertility in females are caused by adhesions (5-10).

Cancer patients usually require retreatment or reoperation [even after adequate treatment, the risk of recurrence of advanced-stage ovarian cancer is as high as $70-90 \%(11)]$. In these patients, adhesions can have an impact on the effects of chemoradiotherapy after surgery and can increase the complexity and risks during surgery, such as prolonged operation time, increased risk of injury, and increased risk of conversion to laparotomy during laparoscopic surgery $(12,13)$. Moreover, $15-20 \%$ of patients who undergo radical hysterectomy due to cervical cancer require radiotherapy after surgery (5), while pelvic adhesions caused by previous abdominal surgery are prone to formation of intestinal adhesions and fixation in the radiation area, which will increase the risks associated with radiation therapy. Adhesions affect the uniform distribution of the radiation dose and causes intestinal fistula, intestinal injury and other consequences and will seriously impact the treatment effects. The degree of intestinal damage is directly related to the total radiation dose, number of treatments, and the dose distribution in the surrounding tissue of the target area (14). For patients with ovarian cancer, intraperitoneal chemotherapy can reduce the risk of death by $21.6 \%$. However, extensive adhesion prevents the drug from spreading in the abdominal cavity, which is a relative contraindication of intraperitoneal chemotherapy due to the limited thorough distribution of intraperitoneal chemotherapy drugs because of severe adhesion (15).

Moreover, the cost of treatments for adhesions results in a heavy economic burden on patients and society. In the United States, abdominal adhesions in inpatients cost \$2.3 billion in 2005. According to several studies, in the United Kingdom, the cost of hospitalization due to adhesions within 2 years after surgeries was $£ 2.42$ million $(16,17)$.

Therefore, preventing postoperative adhesions, improving the quality of patients' lives, and saving medical costs are issues on which every gynecological oncologist must focus.

\section{Formation mechanism and typing of adhesions}

Peritoneal adhesion is the pathological result of repair of peritoneal injury. Adhesion formation can be induced by mechanical or thermal injury, dehydration, incomplete hemostasis, radiation, continuous laparoscopic airflow, prolonged exposure to the light source of the laparoscope during surgery, and by the disease itself (e.g., pelvic inflammatory diseases, endometriosis, infection) (12). After peritoneal injury, mesothelial cell islands are formed locally, mesothelial cells divide and proliferate to repair the wound, and mesothelialization is completed, after which fibrin proteins are degraded to form new peritoneum. After proliferation and maturation, proliferating cells interconnect with each other. After 5-7 days, the basal peritoneum is formed, and the peritoneum recovers normally. However, due to local ischemia or infection during wound repair, fibrin will form a bridge on the surface of two opposing exposed tissues. Most fibrin exudates are transient and are degraded within 72 hours, but the activity of the damageinduced peritoneal fibrin lysis system is inhibited (18), and thus, the fibrin bundles cannot be cleared. Fibroblasts enter the fibrin bridge and release collagen within 3-5 days after surgery. By the 7 th day, small blood vessels form in the fibrin bridge, which leads to the formation of adhesions (Figure 1).

Regarding the grading and classification of adhesions, various international grading standards have been established. Currently, the modified adhesion grading system published by the American Society for Reproductive Medicine (ASRM) (19) is widely used (Table 1).

\section{Prevention of adhesions in gynecological tumor surgeries}

In view of the prevalence of postoperative adhesions, 


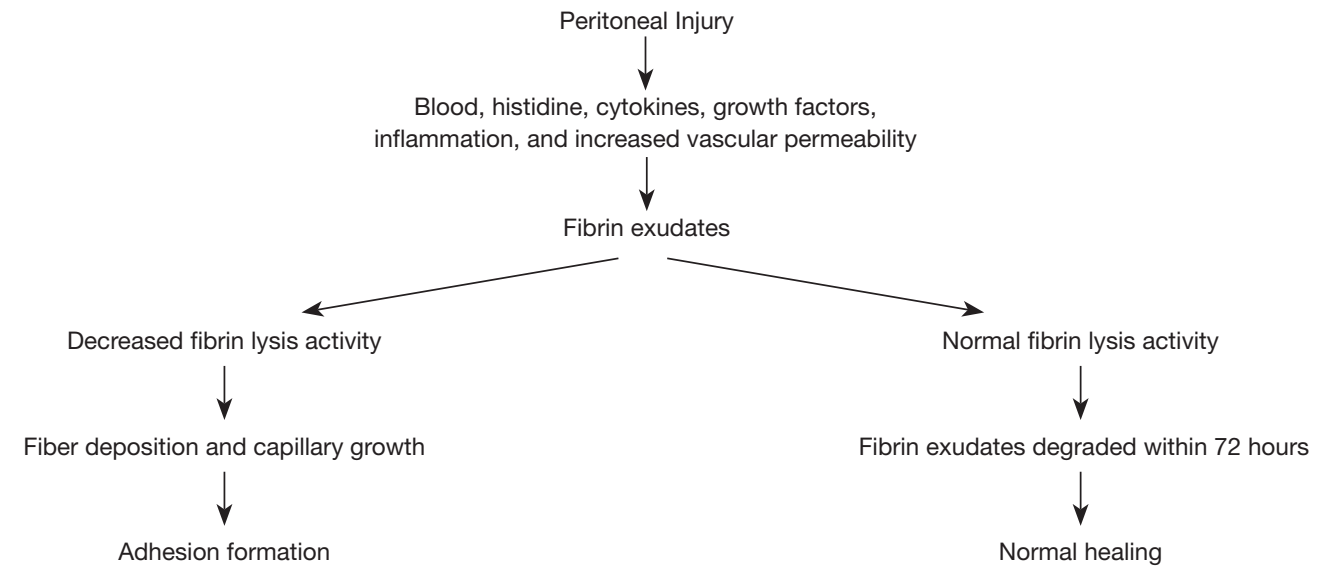

Postoperative adhesion

Practice standardization committee. Gynecological surgery adhesion. Fetil Steril 2013

Figure 1 Mechanism of adhesion formation.

Table 1 Modified American Society for Reproductive Medicine (ASRM) adhesion grading standards

\begin{tabular}{lcc}
\hline The nature and range of adhesions observed during surgery* & Score (points) & Adhesion grading \\
\hline No adhesion & 0 & N/A \\
Membranous, $<25 \%$ & Mild & Mild \\
Membranous, $25-50 \%$ & 3 & Moderate \\
Membranous, $\geq 51 \%$ & 4 & Moderate \\
Dense, $<25 \%$ & 5 & Severe \\
Dense, $25-50 \%$ & 6 & Severe
\end{tabular}

*, the range of adhesions observed during surgery. The range of adhesions observed during surgery refers to the surgeon's evaluation of the adhesions at 15 anatomical locations, including the anterior wall of the uterus, the posterior wall of the uterus, the anterior wall of the abdominal cavity, and the anterior wall of the rectal uterine depression (19).

preoperative informed consent and intraoperative prevention of adhesions must be taken seriously by clinicians. At present, sophisticated surgical techniques and the placement of antiadhesion barriers or agents between damaged tissues are the primary measures that are used to prevent adhesions. Sophisticated surgical techniques are the foundation of strategies to reduce adhesions, and these include considering surgical indications, following the principles of minimally invasive surgeries, gently touching the tissues, and avoiding foreign matter, dehydration, and infection. When a risk of postoperative adhesions is present, auxiliary antiadhesion measures should be considered. Based on principles of safety, effectiveness, ease of use, and cost, antiadhesion barriers can be considered $(20,21)$.

\section{Surgical techniques for the prevention of adhesions in tumor surgeries}

Cervical cancer is the most common gynecological malignancy in China. Early-stage cases are mainly treated by surgery. The scope of surgery includes extensive hysterectomy + pelvic lymph node dissection + abdominal paraaortic lymph node dissection in some cases. This surgery is a standardized and anatomical operation. The surgical features are as follows: (I) posterior peritoneal lymph nodes require routine dissection, and most of the blood vessels behind the pelvic peritoneum, such as the blood vessels and structures in the common ilium, external ilium, and obturator area of the pelvic cavity, 
the entire pelvic segment of the ureter, and partial paraaorta area, need to be anatomically exposed; (II) extensive hysterectomy requires dissection of the space around the cervix, including the separation of the bladder and rectum. Resection of a wide range of para-uterine and vaginal tissues results in significant tissue damage, as well as in the exposure and changes of the pelvic floor structures after surgery, which increases the chances of postoperative tissue wound bleeding, inflammatory infections, and adhesions.

The primary treatment of endometrial cancer is surgery, which is supplemented by comprehensive treatments of radiotherapy, chemotherapy, and hormones. Extrafascial radical hysterectomy combined with bilateral appendectomy is the most basic surgical method for endometrial cancer. Some patients require pelvic lymph node dissection and abdominal paraaortic lymph node dissection (to the level of the renal blood vessels). Deeply infiltrated endometrial cancer results in a large surgical wound, is associated with a relatively difficult surgical procedure, and is prone to bleeding; this cancer has an incidence of postoperative adhesions as high as $56-100 \%(22)$.

Most patients with ovarian cancer are diagnosed at an advanced stage and require open abdominal surgery. Surgical treatments include comprehensive surgery for early-stage patients and tumor cytoreductive surgery for advanced-stage patients. The scope of surgery includes the uterus and appendages, the greater omentum, abdominal paraaortic lymph nodes, bilateral pelvic lymph nodes, tumor-invaded organs, and a large area of tumor-invaded peritoneum. Ovarian cancer surgery results in a large wound surface and a large amount of bleeding and is prone to the formation of adhesions between the intestines and between the abdominal wall and the intestines. After tumor recurrence, tumor cytoreductive surgery is a possibility. Adhesions affect the application and effect of postoperative intraperitoneal chemotherapy, increase the chance of organ damage, cause difficulty in reoperation, and result in a higher probability of postoperative adhesion with a broader range and higher severity (23).

Although surgery is one of the main treatment methods for the three major malignant gynecologic tumors, a large proportion of patients still need chemoradiotherapy after surgery. Patients who undergo malignant tumor surgeries are more prone to adhesions than those who undergo other surgeries. Postoperative radiotherapy or perfusion will increase the chance of adhesions in the surgical area, which will lead to increased adverse complications of radiotherapy or perfusion, as well as an increased probability of organ damage. Therefore, the following characteristics and points should be achieved in gynecological tumor surgeries: (I) use a minimally invasive approach. Minimally invasive refers not only to minimally invasive surgical approaches but also to the avoidance of rough operations during surgery, operations in nonsurgical areas, a large range of electrocoagulation or tearing, and excessive peritoneal loss; (II) minimize the size of the surgical wound, such as by intentionally retaining the peritoneum in the surgical area so that certain areas of the pelvic wall can be peritonealized at the end of the operation; (III) perform sentinel lymph node biopsy in selected appropriate cases to reduce unnecessary peritoneal loss; (IV) avoid bleeding during surgery and strictly perform hemostasis during the surgery; (V) minimize the use of nonabsorbable materials. Rinse the surgical area before the end of the surgery to remove tissue debris and blood clots as much as possible; (VI) pay attention to the principle of sterility and actively prevent infection; (VII) apply antiadhesion products reasonably in areas that are prone to adhesions such as the cervical stump and lymphatic dissection area to further reduce the incidence of adhesions.

\section{Selection of materials and drugs that prevent adbesion}

At present, materials that prevent adhesion are divided into two categories: diaphragm materials and gel/liquid materials. The ideal antiadhesion material should be considered comprehensively for its safety, effectiveness, convenience, and economy, such as a lack of foreign body reaction, absorbability, convenient use in laparoscopic and laparotomic surgeries, and effective prevention of new and regenerative adhesions (12). Five types of widely used antiadhesion materials are shown (Table 2).

\section{Diaphragm materials}

Antiadhesion diaphragm materials are antiadhesion barriers that are easy to use and are currently the most widely studied. According to the surgical location and scope, this material is placed at the location of the wound, stump, and lymphatic dissection area during tumor surgery, where it plays a role in physical insulation and reduces the occurrence of adhesions.

(I) Oxidized regenerated cellulose (ORC) antiadhesion membrane: this is an absorbable antiadhesion barrier composed of ORC (tree pulp extract). After standing for 8 hours, ORC forms a continuous and dense hydrophilic gel on the surface of the tissue and covers the damaged surface. It remains 
Table 2 Materials that prevent adhesion

\begin{tabular}{|c|c|c|c|}
\hline Components & Source and process & Safety & Effectiveness \\
\hline $\begin{array}{l}\text { Oxidized regenerated } \\
\text { cellulose }(\mathrm{ORC})\end{array}$ & $\begin{array}{l}\text { Extracted from natural plant fibers, } \\
\text { oxidative regeneration process }\end{array}$ & $\begin{array}{l}\text { Fully absorbed in } 28 \text { days without } \\
\text { any adverse reactions }\end{array}$ & $\begin{array}{l}\text { Remains intact during the peritoneal } \\
\text { recovery period, } 29 \text { years of clinical } \\
\text { research, good adherence, the first } \\
\text { antiadhesion material approved by the } \\
\text { US FDA }\end{array}$ \\
\hline $\begin{array}{l}\text { DL-polylactic acid } \\
\text { (PLA) }\end{array}$ & $\begin{array}{l}\text { Chemically synthesized DL-polylactic } \\
\text { acid (DL-PLA), produced by extended } \\
\text { pressure processing }\end{array}$ & $\begin{array}{l}\text { The complete degradation } \\
\text { cycle ranges from 8-12 weeks, } \\
\text { no serious adverse reactions are } \\
\text { found, individual patients may } \\
\text { develop mild discomfort or local } \\
\text { soreness }\end{array}$ & Good insulation and adhesiveness \\
\hline \multicolumn{4}{|c|}{ Gel/liquid-type antiadhesion materials } \\
\hline $\begin{array}{l}\text { Sodium hyaluronate } \\
\text { gel }(\mathrm{HA})\end{array}$ & $\begin{array}{l}\text { Cockscomb extract or bacterial } \\
\text { fermentation, uronic acid and } \\
\text { hexosamine condensation, animal } \\
\text { extraction }\end{array}$ & $\begin{array}{l}\text { Nontoxic, immunogenic, } \\
\text { animal-derived products that } \\
\text { may cause adverse reactions in } \\
\text { individual patients }\end{array}$ & $\begin{array}{l}\text { Unstable degradation time ranging } \\
\text { from } 72 \text { hours to } 2 \text { weeks, strong } \\
\text { fluidity, ordinary adhesiveness }\end{array}$ \\
\hline $\begin{array}{l}\text { Carboxymethyl chitin } \\
\text { (Chitosan) }\end{array}$ & $\begin{array}{l}\text { Crustacean shell extract, glucosamine } \\
\text { 14-2-acetamido-a-deoxy-B-, water } \\
\text { as solvent, animal extraction }\end{array}$ & $\begin{array}{l}\text { Nontoxic, individual patients may } \\
\text { have allergic reactions }\end{array}$ & $\begin{array}{l}\text { Unstable degradation time ranging } \\
\text { from } 1-4 \text { weeks, repairing effect, } \\
\text { strong fluidity, ordinary adhesiveness }\end{array}$ \\
\hline
\end{tabular}

intact during the peritoneal recovery cycle (5-7 days) and physically insulates the tissue surface to prevent adhesion formation. After 28 days, ORC is hydrolyzed into carbon dioxide and water, which is completely absorbed by the human body. ORC is convenient to use and operate without the requirement of sutures.

ORC antiadhesion membrane is currently the most widely studied product, as this material has the highest number of clinical literature reports in China and other countries. In randomized controlled clinical trials, this product has been demonstrated to reduce adhesion formation because it reduces the incidence and extent of new and recurrent adhesions after laparoscopic and laparotomic surgeries by $50 \%$ to $60 \%$ (24). A multicenter randomized study showed that ORC antiadhesion membrane (Interceed) could significantly reduce the occurrence and severity of ovarian adhesions after surgery, and the involved area of ovarian surface adhesions was significantly reduced (25). In 2014, the European Society of Human Reproduction and Embryology (ESHRE) guidelines recommended that surgeons apply ORC to prevent adhesion formation after laparoscopic endometriosis surgery (26).

A health economics study evaluated the cost of ORC antiadhesion membrane (Interceed) in the prevention of abdominopelvic adhesions after gynecological and obstetric surgeries. The results showed that the application of this product could significantly reduce the postoperative adhesion rate after ovarian cancer surgeries, reduce the incidence of adhesion complications, and reduce the probability of reoperation, thereby saving patients 2,320 Yuan in medical costs during the 3-year observation period (27).

(II) Sodium hyaluronate-carboxymethyl cellulose antiadhesion membrane: this is a bioabsorbable antiadhesion membrane composed of hyaluronic acid and carboxymethyl cellulose. The material adheres to the wet surface of tissues and turns 
into a near-mature gel 24 hours after placement to physically insulate the tissue surface. It is completely absorbed by the human body within 28 days. This material should not be folded, and therefore, caution is required during its use.

A randomized controlled clinical study showed that, compared with the control group, the incidence of chronic abdominal pain (more than 3 months) was significantly reduced in surgical patients with a chemically modified sodium hyaluronate-carboxymethyl cellulose antiadhesion membrane placed in the abdominopelvic cavity. However, no significant difference was observed between the two groups in the incidence of postoperative small bowel obstruction and postoperative quality of life (28).

(III) DL-polylactic acid (DL-PLA) antiadhesion membrane: this is a kind of absorbable diaphragm material made of polylactic acid fiber. After placement in the human body, it maintains its membrane shape to separate the surgical wound surface from adjacent organs and tissues. The degradation process is divided into hydrolysis and enzymatic metabolism. A randomized controlled clinical study showed that adhesions of the abdominal cavity in patients with polylactic acid antiadhesion membrane placed in the abdominopelvic cavity were significantly milder than those of the control patients who did not receive application of the antiadhesion membrane (29). When using it, attention should be paid to the unsatisfactory antiadhesion effect caused by changes in the membrane covering position due to intestinal peristalsis and other reasons, in which case absorbable sutures can be used for proper fixation.

\section{Gel/liquid materials}

Gel/liquid materials are a type of easy-to-use, degradable, and absorbable antiadhesion material placed on the surface of tissues to reduce the occurrence of adhesions. Such materials are quickly absorbed, while the degradation time is unstable, and the effectiveness of antiadhesion is controversial (12). The gel/liquid materials are substances that flow easily, and thus, the effect of the patient's position change on the efficacy should be noted.

(I) Hyaluronic acid (HA) gel: this is a type of high molecular weight mucopolysaccharide prepared from natural materials. HA gel is both degraded and absorbed. HA gel covers the plasma membrane surface of tissues, physically insulates the wound surface, reduces the formation of adhesions, and avoids the exacerbation of existing adhesions (30). HA gel has a short degradation time due to its unstable molecular structure, but whether HA gel can remain intact and lead to effective insulation during the peritoneal recovery period requires further investigation. Due to different preparation processes, a variety of different HA-based preparations have been developed. HA gel is easy to manipulate and requires a catheter to apply the liquid to the wound surface during laparoscopic or hysteroscopic surgeries.

(II) Carboxymethyl chitin (chitosan): this material is derived from shrimp shell extract and is naturally degraded and absorbed by the human body. It has the biological characteristics of selectively promoting the growth of epithelial cells and endothelial cells and inhibiting the growth of fibroblasts; therefore, chitosan promotes tissue physiological repair, inhibits scar formation, reduces tissue adhesion, and prevents new adhesion formation (31). Chitosan is easy to manipulate, but a catheter is required to apply the liquid to the wound surface during laparoscopic or hysteroscopic surgeries.

\section{Summary}

Abdominopelvic cavity adhesions after gynecological tumor surgeries are extremely common. Adhesions can cause postoperative complications that affect the implementation and effect of chemoradiotherapy after malignant tumor surgeries, and therefore, adhesions must be taken seriously by gynecological oncologists. Before gynecological tumor surgeries, clinicians should fully inform patients about the risk of postoperative adhesion, the impact on subsequent treatments, and preventive measures. Prevention of adhesion is extremely important. Sophisticated tumor surgical techniques are the most important factor in adhesion prevention. The combined application of antiadhesion materials on the basis of sophisticated surgical operations is conducive to the further reduction in the incidence of adhesion. The ideal antiadhesion material should possess the characteristics of safety, effectiveness, convenience, and economy. Appropriate antiadhesion material is selected 
according to the requirements of the surgery, which can comprehensively and effectively prevent the formation of adhesions, decrease medical costs, reduce surgical risks, and improve the quality of life of tumor patients.

\section{Acknowledgments}

None.

\section{Footnote}

Conflicts of Interest: The authors have no conflicts of interest to declare.

Ethical Statement: The authors are accountable for all aspects of the work in ensuring that questions related to the accuracy or integrity of any part of the work are appropriately investigated and resolved.

\section{References}

1. Hirschelmann A, Tchartchian G, Wallwiener M, et al. A review of the problematic adhesion prophylaxis in gynaecological surgery. Arch Gynecol Obstet 2012;285:1089-97.

2. Diamond MP. Incidence of postsurgical adhesions. New York: Springer-Verlag, 2000.

3. Tulandi T, Al-Shahrani A. Adhesion prevention in gynecologic surgery. Curr Opin Obstet Gynecol 2005; 17:395-8.

4. Al-Took S, Platt R, Tulandi T. Adhesion-related smallbowel obstruction after gynecologic operations. Am J Obstet Gynecol 1999;180:313-5.

5. Montz FJ, Holschneider CH, Solh S, et al. Small bowel obstruction following radical hysterectomy: risk factors, incidence, and operative findings. Gynecol Oncol 1994;53:114-20.

6. Farinella E, Cirocchi R, La Mura F, et al. Feasibility of laparoscopy for small bowel obstruction. World J Emerg Surg 2009;4:3.

7. Fevang BT, Fevang J, Lie SA, et al. Long-term prognosis after operation for adhesive small bowel obstruction. Ann Surg 2004;240:193-201.

8. DeWilde RL, Trew G, on behalf of the Expert Adhesions Working Party of the European Society of Gynaecological Endoscopy (ESGE). Postoperative abdominal adhesions and their prevention in gynecological surgery. Gynecol Surg 2007;4:161-8.
9. Stovall TG, Elder RF, Ling FW. Predictors of pelvic adhesions. J Reprod Med 1989;34:345-8.

10. Vercellini P, Somigliana E, Viganò $P$, et al. Chronic pelvic pain in women: etiology, pathogenesis and diagnostic approach. Gynecol Endocrinol 2009;25:149-58.

11. StatBite: Ovarian cancer: risk of recurrence by stage of diagnosis. J Natl Cancer Inst 2009;101:1234.

12. Al-Musawi D, Thompson JN. Adhesion Prevention: State of the Art. Gynecol Endosc 2010;10:123-30.

13. Sokol AI, Chuang K, Milad MP. Risk factors for conversion to laparotomy during gynecologic laparoscopy. J Am Assoc Gynecol Laparosc 2003;10:469-73.

14. Pal N, Geibel J. Radiation Enteritis and Proctitis. Medscape 2017. Available online: https://emedicine. medscape.com/article/197483-overview

15. NCI Clinical Announcement (1/05/06). Intraperitoneal chemotherapy for ovarian cancer.

16. Sikirica V, Bapat $\mathrm{B}$, Candrilli SD, et al. The inpatient burden of abdominal and gynecological adhesiolysis in the US. BMC Surg 2011;11:13.

17. Wilson MS, Menzies D, Knight AD, et al. Demonstrating the clinical and cost effectiveness of adhesion reduction strategies. Colorectal Dis 2002;4:355-60.

18. Diamond MP, El-Mowafi DM. Pelvic adhesions. Surg Technol Int 1998;7:273-83.

19. Mettler L, Hucke J, Bojahr B, et al. A safety and efficacy study of a resorbable hydrogel for reduction of postoperative adhesions following myomectomy. Hum Reprod 2008;23:1093-100.

20. De Wilde RL, Bakkum EA, Brölmann H, et al. Consensus recommendations on adhesions (version 2014) for the ESGE Adhesions Research Working Group (European Society for Gynecological Endoscopy): an expert opinion. Arch Gynecol Obstet 2014;290:581-2.

21. Diamond MP, Freeman ML. Clinical implications of postsurgical adhesions. Hum Reprod Update 2001;7:567-76.

22. Schwartz HE, Blackmore JM. Bioresorbable compositions of carboxy polysaccharide polyether intermolecular complexes and methods for their use in reducing surgical adhesions. San Luis Obispo, CA: FzioMed, Inc., 2000.

23. Fader AN, Rose PG. Role of Surgery in Ovarian Carcinoma. J Clin Oncol 2007;25:2873-83.

24. Practice Committee of the American Society for Reproductive Medicine. Society of Reproductive Surgeons. Pathogenesis, consequences, and control of peritoneal adhesions in gynecologic surgery. Fertil Steril 2007;88:21-6. 
25. Franklin RR. The ovarian adhesion study group. Reduction of ovarian adhesions by the use of Interceed. Ovarian Adhesion Study Group. Obstet Gynecol 1995;86:335-40.

26. Dunselman GA, Vermeulen N, Becker C, et al. ESHRE guideline: management of women with endometriosis. Hum Reprod 2014;29:400-12.

27. Xiang Y, Song L, Jia Y, et al. Cost-Effectiveness Analysis of Absorbable Adhesion Barrier in Preventing Adhesion Formation after Open Gynecologic Surgeries in China. Value Health 2018;21:S144.

28. van der Wal JB, Iordens GI, Vrijland WW, et al. Adhesion

Cite this article as: Lang J, Ma D, Xiang Y, Hua K, Liu K, Pan L, Wang P, Yao S, Zhao F, Cheng W, Cui M, Guo H, Guo R, Hong L, Li P, Liu M, Meng Y, Wang H, Wang J, Wang W, Wu M, Yang X, Zhang J. Chinese expert consensus on the prevention of abdominal pelvic adhesions after gynecological tumor surgeries. Ann Transl Med 2020;8(4):79. doi: $10.21037 /$ atm.2020.02.53 prevention during laparotomy: long-term follow-up of a randomized clinical trial. Ann Surg 2011;253:1118-21.

29. Luo Y, Li Z. Observation on the efficacy of polylactic acid anti-adhesion membrane in the prevention of postoperative adhesion. China Practical Medicine 2012;7:95-6.

30. Metwally M, Watson A, Lilford R, et al. Fluid and pharmacological agents for adhesion prevention after gynaecological surgery. Cochrane Database Syst Rev 2006;(2):CD001298.

31. Diamond MP, Luciano A, Johns DA, et al. Reduction of postoperative adhesions by N,O-carboxymethylchitosan: a pilot study. Fertil Steril 2003;80:631-6. 